\title{
Azole-acridine hybrids as potential enzymatic inhibitors of coronavirus-2 main protease and RNA polymerase by molecular modeling strategy
}

\section{Charles K. Rono}

University of Johannesburg

Banothile C.E. Makhubela ( $\sim$ bmakhubela@uj.ac.za )

University of Johannesburg

\section{Research Article}

Keywords: Coronavirus, Triazoles, Pyrazole, SARS-CoV-2, Main protease, Docking, RNA polymerase

Posted Date: June 16th, 2020

DOI: https://doi.org/10.21203/rs.3.rs-32809/v2

License: (c) (1) This work is licensed under a Creative Commons Attribution 4.0 International License.

Read Full License 


\section{Abstract}

SARS-CoV-2 has been identified as the cause of the current outbreak of coronavirus disease (COVID-19). As part of the efforts to develop potential drugs with promise for clinical use, a molecular docking study on azole (triazole and pyrazole) based molecules on the main protease $\mathrm{M}^{\text {pro }}$ and RNA polymerase was conducted, as possible inhibitors that could be elected for further experimental bioassays. Autodock has been employed to identify azole derivatives 1-6 preferred conformations in the active site of the enzyme and to estimate their binding affinities to the protease and RNA polymerase targets. From the molecular docking strategy, these new azole compounds though nonpeptides in nature display possible inhibition of $\mathrm{M}^{\text {pro }}$ activity with comparable affinities $(-4.7 \mathrm{kcal} / \mathrm{mol}$ to $-6.5 \mathrm{kcal} / \mathrm{mol})$ to the recently reported peptidelike inhibitors such as a-ketoamide inhibitor $13 \mathrm{~b}(-5.0 \mathrm{k} / \mathrm{cal} / \mathrm{mol})$. They also exhibit improved binding affinities to RNA polymerase $(-6.3$ to $-7.1 \mathrm{kcal} / \mathrm{mol})$ comparable to remdesivir $(-6.6 \mathrm{kcal} / \mathrm{mol})$. Based on the observed binding energies, these compounds may possess anti-coronavirus bioactivity through inhibition of the virus main protease as well as RNA polymerase activities in living cells.

\section{Introduction}

Coronavirus disease (Covid-19) outbreak is currently a pandemic as declared by WHO in March $2020 .{ }^{1}$ It is caused by the new severe acute respiratory syndrome coronavirus-2, SARS-CoV-2. Owing to the novelty of the coronavirus, neither vaccines nor defined treatments for coronavirus infections are available at the moment which has contributed to increased efforts to control its global spread. There were over 4.4 million globally confirmed cases of COVID-19 as of $16^{\text {th }}$ May 2020 since the first case was reported in December 2019 in Wuhan City, China. ${ }^{2}$ The successful isolation and genomic sequence of SARS-CoV-2 has been critical in understanding the novel corona virus, identification of diagnostic kits and establishment of the key pathways involved in the development and spread of the virus. The recent breakthrough in identification of the virus pathway of action including receptors and the viral proteins determinants in transmission has provided a significant advancement in the current search for treatment. ${ }^{3-6}$ Main protease $\left(\mathrm{M}^{\text {pro }}\right)$ has been established as one of the key enzymes involved in the viral replication and maturation of the coronavirus proteome. ${ }^{4,7-9}$ It plays a fundamental role in the processing of two large polyproteins (PP1a and PP1b) which are required for the virus to mature and cause infections. $\mathrm{M}^{\text {pro }}$ has at least

11 cleavage sites on the polyprotein and a unique cleavage site sequence Leu-GIn $\downarrow$ (Ser,Ala,Gly)) recognition, and is thus a vital molecular target in the development of anti-coronavirus therapy. ${ }^{4,10}$ On the other hand, RNA polymerase mediates the replication of the SARS-CoV-2 virus through a complex of non-structural proteins (nsp) for the virus. ${ }^{6,11-12}$ Inhibition of the RNA-dependent RNA polymerase (RdRp) activity will rationally hinder binding of the template and reduce/limit its replication process, and is therefore a fundamental molecular target for antiviral drugs. As part of our contribution to the scientific efforts to develop novel therapeutic agents against coronavirus (CoV-2), we sought to evaluate new azole based molecules recently developed in our laboratory ${ }^{13}$ for their possible anti-coronavirus activity 
through molecular docking strategy. Herein, we provide a discussion on the bioinformatics of the triazole and pyrazole derivatives containing acridine frameworks as possible inhibitors of SARS-CoV-2 $\mathrm{M}^{\text {pro }}$ and RNA-dependent RNA-polymerase (RdRp). An insight into the kind of conformations adapted by the new azole hybrids in the active site of $\mathrm{M}^{\text {pro }}$ and RdRp, binding affinities and key interactions has been provided through computational methods, mainly molecular docking.

\section{Methodology}

Ligand and protein preparation For docking studies, the ligands were converted into autodock input file using Open Babel. ${ }^{14}$ Ligand preparation involved geometry optimization, energy minimization and assignment of partial charges; a prerequisite file format for autodock input. The crystal structure of the protein of (un)liganded main protease, $M^{\text {pro }}$ (ID: 6lu7), main protease $\mathrm{M}^{\text {pro }}$ co-crystallized with aketoamide inhibitor $13 b$ (6Y2F/6Y2G) for binding site identification and SARS-CoV-2 RNA-dependent RNA-polymerase, RdRp, (ID: 7BV1; for apoRdRp, and ID: 7BV2; for the Remdesivir liganded-RdRp in complex with a template-primer) were obtained from the protein data bank (https://www.rcsb.org/).. Each of these molecular targets was transformed into docking input files using autodock program.

Docking and analysis: Autodock files for the ligands and the proteins were transferred to the autodock module in PyRx software. ${ }^{15-16}$ Docking grid parameters (Table S1) were defined based on the residues of the binding site/pockets of the reported inhibitors co-crystallized with protease $\mathrm{M}^{\text {pro }} 4,17$ and RdRp (remdesivir) in the crystal structures. ${ }^{6}$ Docking studies and analysis of the binding poses/affinities were performed using autodock vina program in PyRx software. ${ }^{15}$ The binding conformations of the ligands in the active site of the SARS-CoV-2 $\mathrm{M}^{\text {pro }}$ and RdRp, and their corresponding protein-ligand interactions were visualized and processed using Discovery Studio 4.118 or PyMol program. ${ }^{19}$

\section{Results And Discussions}

\section{Chemistry of the compounds}

The compounds evaluated in this study include azole-acridine molecular hybrids of triazole and pyrazole containing acridines designed rationally for targeted therapy with nucleic acid as one of the molecular targets. Figure 1 shows the molecular triazole and pyrazole hybrids elected for inhibition of main protease SARS-CoV M ${ }^{\text {pro }}$ by molecular docking. The synthesis of the acridine based triazole hybrids has been reported previously with 1,3-dipolar cycloaddition as a key reaction in the triazole heterocycle synthesis. ${ }^{13} \mathrm{~A}$ convergent reaction between 3-phenylpyrazole and acridine in the presence of sodium hydride and 2-bromoethylamine hydrobromide salt afforded the ethyl linked acridine based pyrazole hybrid 6 analogous to triazole 2 . 


\section{Molecular docking studies: To determine the binding affinity, $\mathrm{BE}$, of the azole derivatives}

To probe the potential of the synthesized compounds for anti-coronavirus activity, a docking strategy was employed to not only determine their enzymatic binding affinities and preferred conformations on the SARS-CoV-2 main protease $\left(\mathrm{M}^{\text {pro }}\right)$ and RdRp but also to identify the key interactions involved in their binding sites. Notably, the selected binding site was derived from the liganded crystal structures of SARSCoV-2 M pro (PDBID: 6lu7) which co-crystallized with peptide-like compounds as candidate inhibitors. ${ }^{4}, 17$ Experimentally determined a-ketoamide inhibitor ${ }^{20} 13 b$ was used as a reference inhibitor to corroborate the methodology used in this docking study as well as for comparative assessment of the potential of these new compounds as potential inhibitors of SARS-CoV-2 $\mathrm{M}^{\text {pro }}$ through their binding affinities. For $\mathrm{RdRp}$, the binding conformations and affinities of the ligands were determined relative to the crystallographic site of remdesivir. ${ }^{6}$

\section{Table 1: Binding affinities of the azole ligands with Mpro protease (PDB ID: 6lu7) and Rd-RNA polymerase (PDB ID: 7bv1).}

\begin{tabular}{|c|c|c|}
\hline \multirow{2}{*}{ Ligands } & \multicolumn{2}{|c|}{ Binding Affinity (kcal/mol) } \\
\hline & $M^{\text {pro }} 6 l u 7$ & RdRp 7bv1 \\
\hline 1 & -5.5 & -6.6 \\
\hline 2 & -6.1 & -7.1 \\
\hline 3 & -5.8 & -6.3 \\
\hline 4 & -6.5 & -6.8 \\
\hline 5 & -4.7 & -5.6 \\
\hline 6 & -6.5 & -7.1 \\
\hline $\mathrm{CQ}_{21}$ & -4.9 & -5.4 \\
\hline $\mathrm{HCQ}^{22}$ & -5.3 & -6.1 \\
\hline Remdesivir $^{6,23}$ & -5.5 & -6.6 \\
\hline$\alpha \_$ketoamide $13 b^{4}$ & -5.0 & -7.6 \\
\hline
\end{tabular}

In addition, other drugs such as chloroquine (CQ), ${ }^{21}$ hydroxychloroquine (HCQ) ${ }^{22}$ and remdesivir, 6,23 which are currently being used or under clinical trials were included in the docking study for comparative purposes. Table 1 shows the binding affinities of the most preferred binding poses obtained for each of the compounds evaluated in this study based on the docking scoring function.

All the compounds $1-6$ displayed binding affinities (in the range of $-4.7 \mathrm{kcal} / \mathrm{mol}$ to $-6.5 \mathrm{kcal} / \mathrm{mol}$ ), CQ $(-4.9 \mathrm{kcal} / \mathrm{mol}), \mathrm{HCQ}(-5.3 \mathrm{kcal} / \mathrm{mol})$ and remdesivir $(-5.5 \mathrm{kcal} / \mathrm{mol})$ comparable to that of a- ketoamide inhibitor $13 b(B . E=-5.0 \mathrm{kcal} / \mathrm{mol})$. Hybrids based on triazole exhibited binding affinities, B.E, in the range 
of $-4.7 \mathrm{kcal} / \mathrm{mol}$ to $-6.5 \mathrm{kcal} / \mathrm{mol}$ on docking to the active site of SARS- CoV- $2 \mathrm{M}^{\text {pro }}$ protease as defined by co-crystallized peptide-like inhibitors (B.E of $13 \mathrm{~b}=-5.0 \mathrm{kcal} / \mathrm{mol}) .{ }^{4,17}$

As observed in Figure 2 - Figure 4, the docking models reveal hydrogen bonds are among the key interactions with residues in the active site of the protease which include Thr24/26/45, His41/164, GIn189, Met49, Gly143 and Cys145 - all of which are located in domain I and II of SARS-CoV-2 Mpro protease. ${ }^{4,20}$

The presence of acridine framework in the triazole derivatives $1-4(-5.5 \mathrm{kcal} / \mathrm{mol}$ to $-6.5 \mathrm{kscal} / \mathrm{mol})$ led to improved binding affinities relative to non-acridine based triazole $5(-4.7 \mathrm{kcal} / \mathrm{mol})$, possibly due to improved hydrophobic interactions between the acridine moiety and the amino acid residues in the binding pocket of SARS-CoV-2 $\mathrm{M}^{\text {pro }}$ (Figure 2 and Figure 3 ).

It is notable that for acridine containing azole hybrids $1-4$ and 6 , the presence of acridine moiety orients the molecule in such a way that the 9-amino group forms at least two key $\mathrm{H}$-bonds with thiol and imidazole groups of Cys 145 and His 41 amino acid residues, respectively. In a similar fashion, the two amino acids (Cys145 \& His41) contribute to the interaction of a-ketoamide inhibitor 13b with SARS-CoV$2 \mathrm{M}^{\text {pro }}$ though through reversible covalent bond. Importantly, Cys145 and His41 residues are catalytic dyads responsible for the catalytic actions of SARS-CoV-2 $\mathrm{M}^{\text {pro }}$ as well as other SARS-CoV protease. ${ }^{4,10}$, ${ }^{20}$ Variations of the groups anchored on the azole moiety has some influence on the binding affinity of the azole hybrid and thus allowed preliminary evaluation of the structure activity relationships of these set of azole hybrids.

Hydrophobic interactions are significant in the binding of the azole tail to the Thr24/26 binding pocket, with the phenyl moiety interactions being greater than that of cyclopropyl and hydroxymethyl group $(\mathrm{H}-$ bond) in that order. While non-acridine based triazole 5 assumes an orientation in which the triazole moiety behaves as the tail end as in the other azole derivatives, the $t$-butyl carbarmate ( $t$-boc) moiety forms $\mathrm{H}$-bond with Cys145 but not His41. This reduced hydrogen bonding with the catalytic dyad of SARS-CoV-2 $\mathrm{M}^{\text {pro }}$ could account for the observed relatively low binding affinity $(-4.7 \mathrm{kcal} / \mathrm{mol})$ of triazole 5. It therefore implies that these azoles may exert their anti-SARS-CoV- $2 \mathrm{M}^{\text {pro }}$ inhibition through disruption of Cys145--His41 interactions of the catalytic dyad.

In a similar fashion to the inhibition activity against protease $M^{\text {pro }}$, the azole compounds $1-6$ have exhibited good binding affinities to SARS-CoV-2 RNA-dependent RNA polymerase (RdRp) in the range of -5.6 to $-7.1 \mathrm{kcal} / \mathrm{mol}$ (Table 1). All the azole-acridine hybrids $1-4$ and 6 displayed good affinities for RdRp active site comparable to that of remdesivir $(-6.6 \mathrm{kcal} / \mathrm{mol})$ with binding energies below -6.3 $\mathrm{kcal} / \mathrm{mol}$. Their binding affinities are interestingly better than that of CQ $(-5.4 \mathrm{kcal} / \mathrm{mol})$ and HCQ $(-6.1$ $\mathrm{kcal} / \mathrm{mol}$ ). Also, azole-acridine hybrids exhibit better affinities for RdRp active site than the non-acridine containing triazole $5(-5.6 \mathrm{kcal} / \mathrm{mol})$ implying that the acridine moiety plays an important role in the activity of the azole derivatives. The presence of the phenyl group on the triazole and pyrazole moieties appears to improve the binding affinities of the azole- acridine hybrids $(2,4$ and 6$)$ as evidenced by 
binding energies below $-6.6 \mathrm{kcal} / \mathrm{mol}$ (Table 1) in comparison azole-acridines $1(-6.6 \mathrm{kcal} / \mathrm{mol})$ and 3 $(-6.3 \mathrm{kcal} / \mathrm{mol})$ with hydroxymethyl and cyclopropyl groups respectively. Besides, the chloro and methoxy groups on the acridine framework contribute to improved binding affinities of the azole-acridine evidenced by favorable binding of azole-acridine 2 and $6(-7.1 \mathrm{kcal} / \mathrm{mol}$ each) compared to $4(-6.8$ $\mathrm{kcal} / \mathrm{mol})$. While the acridine framework in each of the azole-acridine hybrids occupy the same pocket in the RdRp, it is interesting to note that the preferred conformation of azole-acridine 1 adapts an opposite orientation to that of the other azole-acridines 2, 3, 4 and 6 (Figure S1) in the RdRp-ligand complexes. This could be attributed to the polarity of the binding pocket of the triazole moiety in 1 as evidenced by hydrogen bonding of the hydroxyl group with amino acid residues Asp618/760/761 as compared to the hydrophobic binding pocket of the phenyl group of azole- acridines 2, 4, and 6 (Figures S1 and S3). The observed better binding affinities suggest that the azole-acridine hybrids may disrupt the activities of RNA-dependent RNA-polymerase by forming significant local interactions with the active site limiting the replication activity of the viral RNA polymerase. ${ }^{6}$

The results from these docking strategies provide preliminary evidence to support the rationale for anticoronavirus assays of these reported azole compounds developed from our laboratory ${ }^{13}$ and suggest further study on their in vitro and/or in vivo assays are necessary to validate these models.

The cheminformatics described herein imply that these compounds may hold promise for optimization as lead compounds and possibly in the development of drug candidates for development into anticoronavirus agents.

\section{Conclusion}

From the computed binding affinities, these recently synthesized azole compounds exhibit comparable binding affinities $(-4.7$ to $-6.5 \mathrm{kcal} / \mathrm{mol})$ to the SARS-CoV-2 $\mathrm{M}^{\text {pro }}$ inhibitors $(-5.0 \mathrm{kcal} / \mathrm{mol}$ for aketoamide $13 \mathrm{~b})$ as well as drugs in clinical trials $(-4.9 \mathrm{kcal} / \mathrm{mol}$ for CQ, $-5.3 \mathrm{kcal} / \mathrm{mol}$ for HCQ and -5.5 $\mathrm{kcal} / \mathrm{mol}$ for remdesivir). Thus they may locally inhibit the activities of the main protease and RNAdependent RNA polymerase in vitro/vivo. Molecular dynamics studies are currently in progress to simulate and estimate the time-dependent binding variations as well as the role played by the solvents in the dynamics of the molecules. Therefore, there is need for further work on bioassays of these nonpeptide compounds to investigate their in vitro/in vivo anti-coronavirus activities. Experimental enzyme inhibition studies of these compounds on SARS-CoV- $2 M^{\text {pro }}$ and RdRp should also be undertaken as part of the bioassays in order to establish whether the inhibition of the SARS-CoV-2 Mpro and RdRp could be responsible for the outcome of anti-coronavirus assays.

\section{References}

1. WHO, WHO Director-General's opening remarks at the media briefing on COVID-19-11 March 2020. 2020. 
2. WHO, Coronavirus disease 2019 (COVID-19): Situation Report - 117. 2020.

3. Wu, C.; Liu, Y.; Yang, ; Zhang, P.; Zhong, W.; Wang, Y.; Wang, Q.; Xu, Y.; Li, M.; Li, X.; Zheng, M.; Chen, L.; $\mathrm{Li}, \mathrm{H}$. ., Analysis of therapeutic targets for SARS-CoV-2 and discovery of potential drugs by computational methods. Acta Pharmaceutica Sinica B 2020.

4. Zhang, L.; Lin, ; Sun, X.; Curth, U.; Drosten, C.; Sauerhering, L.; Becker, S.; Rox, K.; Hilgenfeld, R., Crystal structure of SARS-CoV-2 main protease provides a basis for design of improved a-ketoamide inhibitors. Science. 2020, eabb3405.

5. Wrapp, D.; Wang, ; Corbett, K. S.; Goldsmith, J. A.; Hsieh, C.-L.; Abiona, O.; Graham, S.; McLellan, J. S., Cryo-EM structure of the 2019-nCoV spike in the prefusion conformation. Science. 2020, 367 (6483), 1260-1263.

6. Yin, W.; Mao, C.; Luan, X.; Shen, D.-D.; Shen, ; Su, H.; Wang, X.; Zhou, F.; Zhao, W.; Gao, M., Structural basis for inhibition of the RNA-dependent RNA polymerase from SARS-CoV- 2 by remdesivir. Science. 2020.

7. Kaeppler, U.; Stiefl, N.; Schiller, M.; Vicik, ; Breuning, A.; Schmitz, W.; Rupprecht, D.; Schmuck, C.; Baumann, K.; Ziebuhr, J.; Schirmeister, T., A New Lead for Nonpeptidic Active- Site-Directed Inhibitors of the Severe Acute Respiratory Syndrome Coronavirus Main Protease Discovered by a Combination of Screening and Docking Methods. J. Med. Chem. 2005, 48 (22), 6832-6842.

8. Lu, I. L.; Mahindroo, N.; Liang, -H.; Peng, Y.-H.; Kuo, C.-J.; Tsai, K.-C.; Hsieh, H.-P.; Chao, Y.-S.; Wu, S.-Y., Structure-Based Drug Design and Structural Biology Study of Novel Nonpeptide Inhibitors of Severe Acute Respiratory Syndrome Coronavirus Main Protease. J. Med. Chem. 2006, 49 (17), 5154-5161.

9. Wang, H.; Xue, ; Yang, H.; Chen, C., Recent progress in the discovery of inhibitors targeting coronavirus proteases. Virologica Sinica 2016, 31 (1), 24-30.

10. Anand, K.; Palm, G. J.; Mesters, J. R.; Siddell, S. G.; Ziebuhr, J.; Hilgenfeld, R., Structure of coronavirus main proteinase reveals combination of a chymotrypsin fold with an extra a-helical domain. The EMBO Journal 2002, 21 (13), 3213-3224.

11. Ahn, D.-G.; Choi, J.-K.; Taylor, R.; Oh, J.-W., Biochemical characterization of a recombinant SARS coronavirus nsp12 RNA-dependent RNA polymerase capable of copying viral RNA templates. Arch. Virol 2012, 157 (11), 2095-2104.

12. te Velthuis, J. W.; Arnold, J. J.; Cameron, C. E.; van den Worm, S. H. E.; Snijder, E. J., The RNA polymerase activity of SARS-coronavirus nsp12 is primer dependent. Nucleic Acids Res. 2009, 38 (1), 203-214.

13. Rono, C. K.; Darkwa, J.; Meyer, D.; Makhubela, B. C., A Novel Series of N-aryltriazole and Nacridinyltriazole Hybrids as Potential Anticancer Agents. Org. Synth. 2019, 16 (6), 900-912.

14. O'Boyle, N. M.; Banck, ; James, C. A.; Morley, C.; Vandermeersch, T.; Hutchison, G. R., Open Babel: An open chemical toolbox. J. Cheminform. 2011, 3 (1), 33.

15. Trott, O.; Olson, J., AutoDock Vina: improving the speed and accuracy of docking with a new scoring function, efficient optimization, and multithreading. J. Comput. Chem. 2010, 31 (2), 455-461. 
16. Dallakyan, S.; Olson, A. , Small-molecule library screening by docking with PyRx. Methods Mol Biol 2015, 1263, 243-50.

17. Jin, ; Du, X.; Xu, Y.; Deng, Y.; Liu, M.; Zhao, Y.; Zhang, B.; Li, X.; Zhang, L.; Peng, C.; Duan, Y.; Yu, J.; Wang, L.; Yang, K.; Liu, F.; Jiang, R.; Yang, X.; You, T.; Liu, X.; Yang, X.; Bai, F.; Liu, H.; Liu, X.; Guddat, L. W.; Xu, W.; Xiao, G.; Qin, C.; Shi, Z.; Jiang, H.; Rao, Z.; Yang, H., Structure of Mpro from COVID-19 virus and discovery of its inhibitors. bioRxiv 2020, 2020.02.26.964882.

18. BIOVIA, D. S., Discovery Studio Modeling Environment, Release 2017. San Diego: Dassault Systèmes 2016.

19. DeLano, W. L., The PyMOL Molecular Graphics System. Delano Scientific, San Carlos, CaliforniaUSA. 2002.

20. Zhang, L.; Lin, ; Kusov, Y.; Nian, Y.; Ma, Q.; Wang, J.; von Brunn, A.; Leyssen, P.; Lanko, K.; Neyts, J.; de Wilde, A.; Snijder, E. J.; Liu, H.; Hilgenfeld, R., a-Ketoamides as Broad-Spectrum Inhibitors of Coronavirus and Enterovirus Replication: Structure-Based Design, Synthesis, and Activity Assessment. J. Med. Chem. 2020.

21. Devaux, C. A.; Rolain, J.-M.; Colson, P.; Raoult, D., New insights on the antiviral effects of chloroquine against coronavirus: what to expect for COVID-19? J. Antimicrob. Agents. 2020, 105938.

22. Biot, C.; Daher, ; Chavain, N.; Fandeur, T.; Khalife, J.; Dive, D.; De Clercq, E., Design and Synthesis of Hydroxyferroquine Derivatives with Antimalarial and Antiviral Activities. J. Med. Chem. 2006, 49 (9), 2845-2849.

23. Sheahan, T. P.; Sims, C.; Graham, R. L.; Menachery, V. D.; Gralinski, L. E.; Case, J. B.; Leist, S. R.; Pyrc, K.; Feng, J. Y.; Trantcheva, I.; Bannister, R.; Park, Y.; Babusis, D.; Clarke, O.; Mackman, R. L.; Spahn, J. E.; Palmiotti, C. A.; Siegel, D.; Ray, A. S.; Cihlar, T.; Jordan, R.; Denison, M. R.; Baric, R. S., Broadspectrum antiviral GS-5734 inhibits both epidemic and zoonotic coronaviruses. Sci. Transl. Med. 2017, 9 (396), eaal3653.

\section{Declarations}

\section{Author Contributions}

All authors contributed equally to the article

\section{Additional Information}

Competing Interests: The authors declare no competing interests.

\section{Data Availability}

The data analyzed have been included in this article and the support information. 


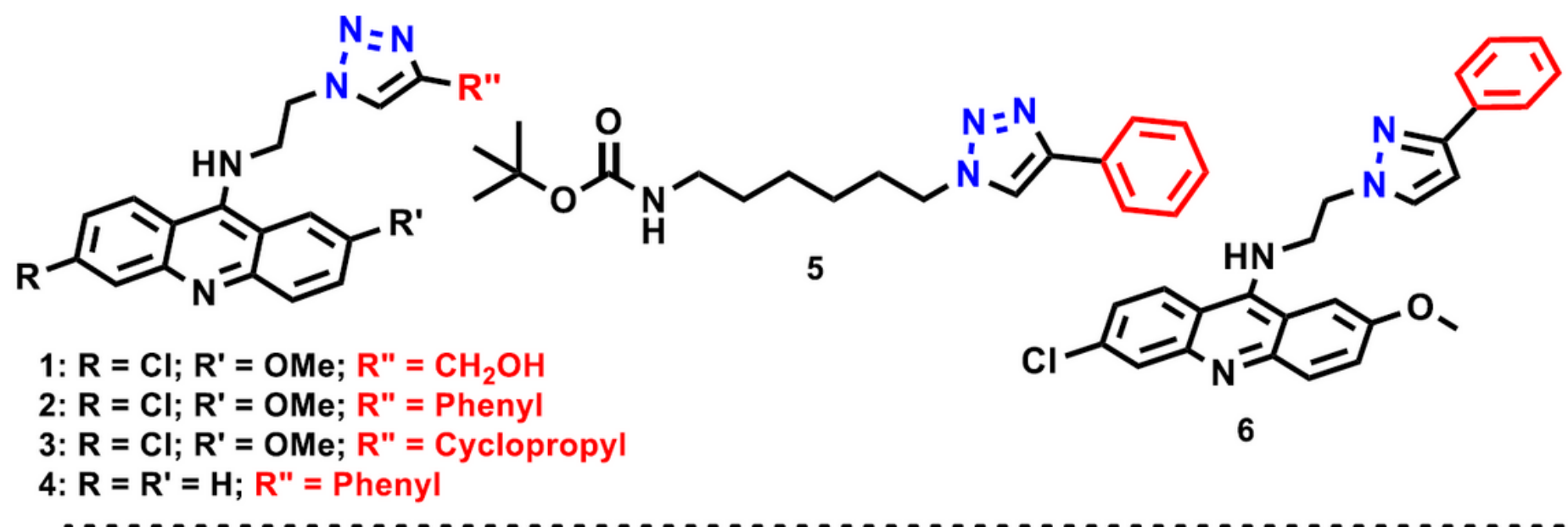

Figure 1

Triazole and pyrazole derivatives selected for SARS-CoV-2 main protease (Mpro) and RNA- dependent RNA polymerase (RdRp) inhibition docking studies.13

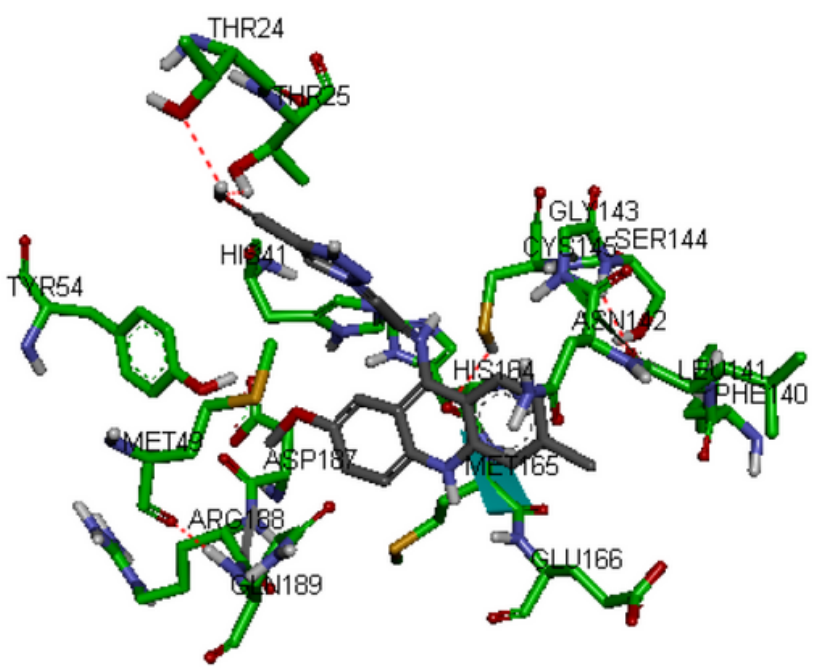

(a)

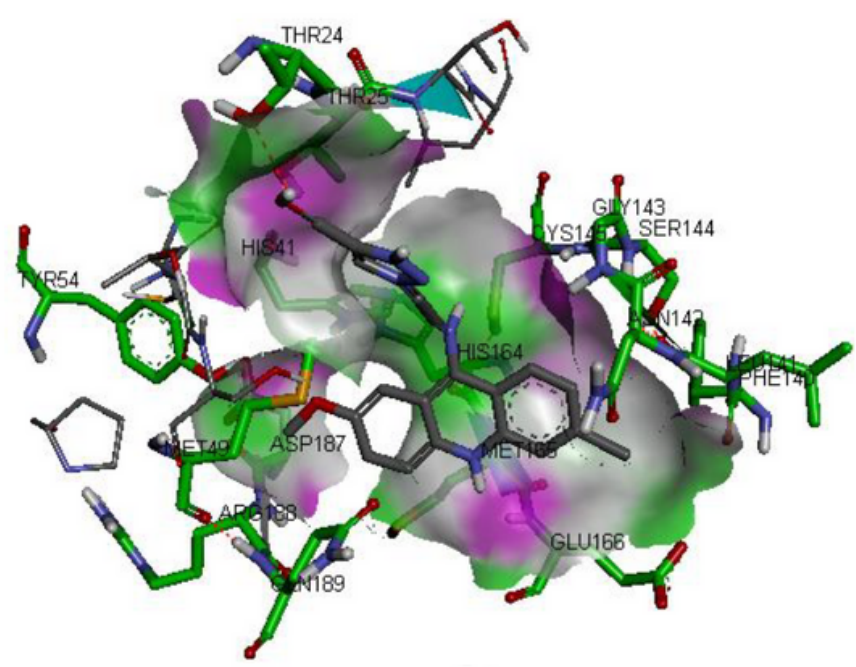

(b)

Figure 2

Protease Mpro_triazacridine 1 complex showing the amino acid residues in (a) the active site 4 and (b) the active site surface. Hydrogen bond interactions between the triazacridine 1 and the amino acid residues are shown in dashed lines. 

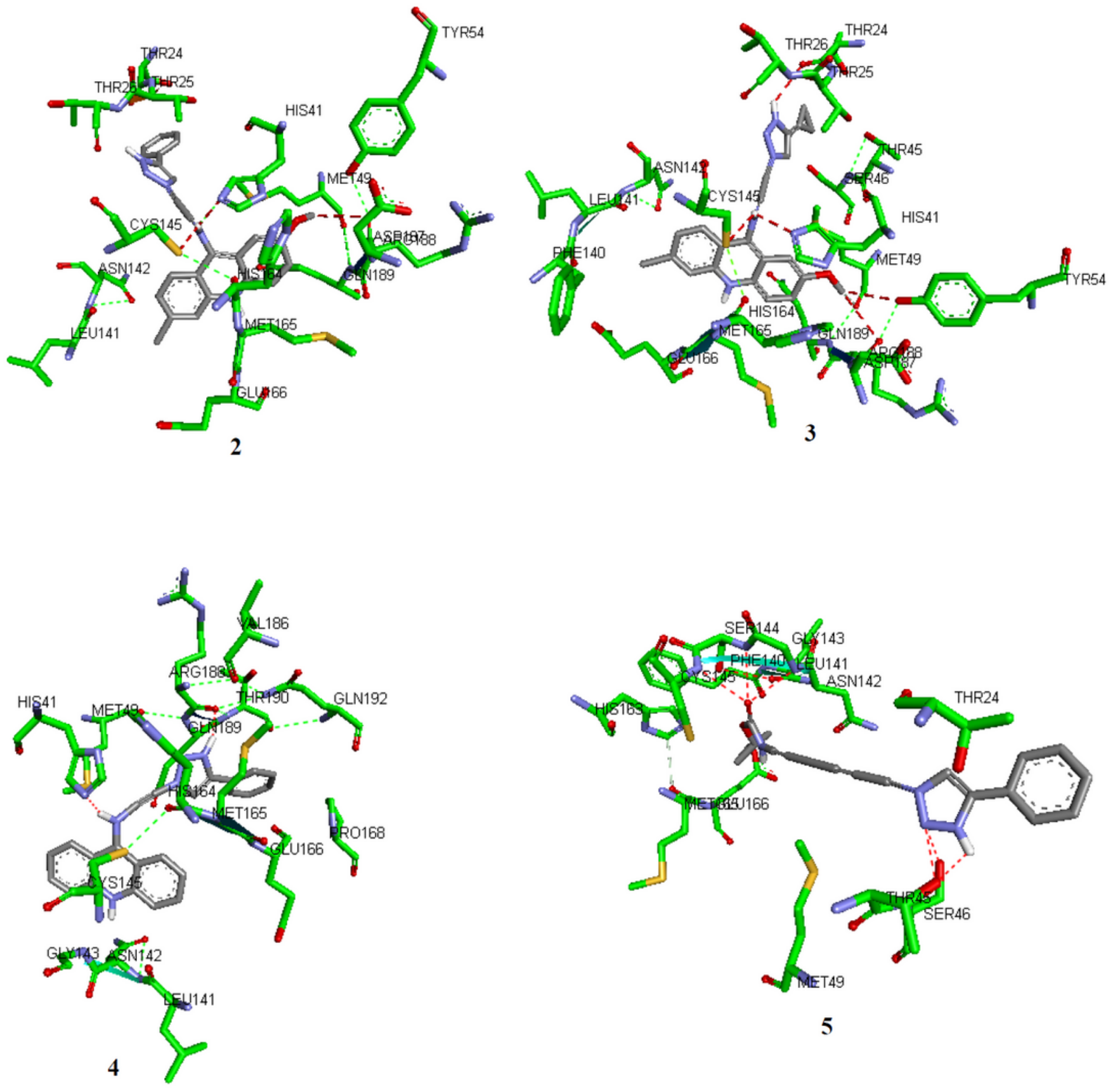

5

Figure 3

Preferred binding conformations of triazoles 2-5 in the binding site of 6lu7 Mpro protease of SARS- CoV2. Hydrogen bonds are shown as dashed bonds from the participating atom in the ligand to the binding pocket amino acid residues. 

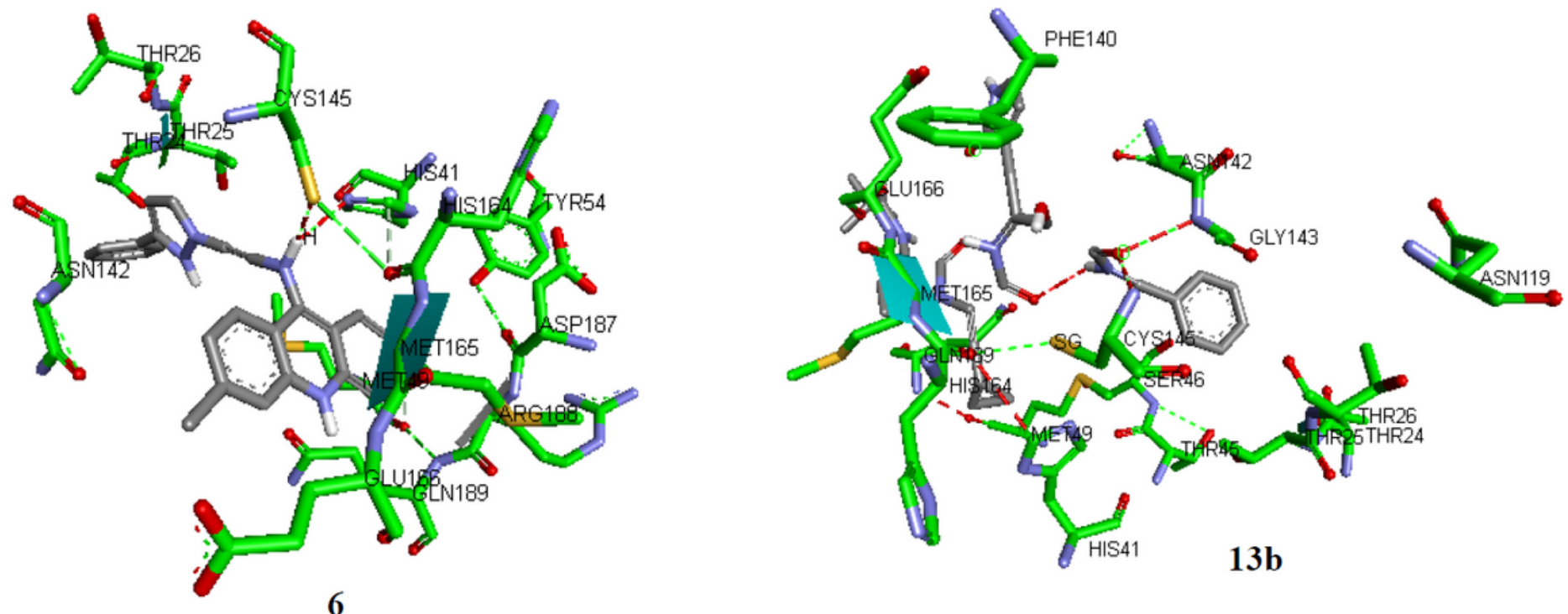

Figure 4

Binding conformations of pyrazole 6 and a-ketoamide inhibitor 13b docked on the binding pocket of SARS-CoV-2 main protease Mpro. Hydrogen-bonds are shown as dashed bonds.

\section{Supplementary Files}

This is a list of supplementary files associated with this preprint. Click to download.

- BMManuscriptSupportlnfos.pdf 